\title{
Performance Optimization of Spin-Torque Microwave Detectors with Material and Operational Parameters
}

\author{
X. Li, ${ }^{1}$ Y. Zhou, ${ }^{2,3}$ and Philip W. T. Pong ${ }^{1}$ \\ ${ }^{1}$ Department of Electrical and Electronic Engineering, The University of Hong Kong, Hong Kong \\ ${ }^{2}$ Department of Physics, The University of Hong Kong, Hong Kong \\ ${ }^{3}$ School of Electronics Science and Engineering, Nanjing University, Nanjing 210093, China
}

Correspondence should be addressed to Y. Zhou; yanzhou@hku.hk and Philip W. T. Pong; ppong@eee.hku.hk

Received 1 February 2016; Accepted 30 May 2016

Academic Editor: Oded Millo

Copyright (C) $2016 \mathrm{X}$. Li et al. This is an open access article distributed under the Creative Commons Attribution License, which permits unrestricted use, distribution, and reproduction in any medium, provided the original work is properly cited.

\begin{abstract}
Sensitivity, bandwidth, and noise equivalent power (NEP) are important indicators of the performance of microwave detectors. The previous reports on spin-torque microwave detectors (STMDs) have proposed various approaches to increase the sensitivity. However, the effects of these methods on the other two indicators remain unclear. In this work, macrospin simulation is developed to evaluate how the performance can be optimized through changing the material (tilt angle of reference-layer magnetization) and operational parameters (the direction of magnetic field and working temperature). The study on the effect of magnetic field reveals that the driving force behind the performance tuning is the effective field and the equilibrium angle between the magnetization of the free layer and that of the reference layer. The material that offers the optimal tilt angle in reference-layer magnetization is determined. The sensitivity can be further increased by changing the direction of the applied magnetic field and the operation temperature. Although the optimized sensitivity is accompanied by a reduction in bandwidth or an increase in NEP, a balance among these performance indicators can be reached through optimal tuning of the corresponding influencing parameters.
\end{abstract}

\section{Introduction}

Spintronics is an emerging field of research on the interaction between the spin of electrons and the magnetization of magnetic materials. The discovery of giant magnetoresistance (GMR) effect, for which Fert [1] and Grünberg [2] were awarded the 2007 Nobel Prize, has proved that the spin of electrons can be polarized by the magnetization of magnetic materials. Meanwhile, the spin current is also capable of altering the magnetization of the ferromagnetic material [3, 4] through the spin-transfer torque (STT) effect $[5,6]$. This observation has led to the development of spin-torque oscillators $[7,8]$, which can change direct current into frequencytunable microwave signal. It was later shown that when microwave current flows through a magnetic tunnel junction (MTJ) nanopillar, a rectified DC voltage $\left(V_{\text {mix }}\right)$ is generated, revealing its potential application as spin-torque microwave detectors (STMDs) [9].

Sensitivity, bandwidth, and noise equivalent power (NEP) are three important performance indicators for STMDs.
Through adjusting the magnitude of the magnetic field $(H)$, the working frequency of STMDs $\left(f_{P}\right)$ can be tuned to match that of the incident microwave to achieve the largest DC output. The sensitivity is defined as the ratio of the peak DC voltage to the incident microwave power. The bandwidth is an evaluation of the range of achievable $f_{P}$ within a certain range of $H$. NEP, on the other hand, is a parameter reflecting the minimum detection power, defined by noise power spectrum density over sensitivity. Although the three indicators are all important for a microwave detector, most of the scientific efforts are devoted to the optimization of sensitivity since competitive sensitivity is the prerequisite for industrial application [10]. The previous publications reported increased sensitivity through applying DC bias [11], optimizing the orientation of in-plane (IP) and out-of-plane (OOP) magnetic field $[10,12,13]$, and adjusting the IP shift angle of reference-layer magnetization [14]. All these optimizations have resulted in the record high sensitivity of over $14,000 \mathrm{mV} / \mathrm{mW}$ under tilted magnetic field [15] and $75,400 \mathrm{mV} / \mathrm{mW}$ under zero magnetic field [16]. Although these reported sensitivities far 

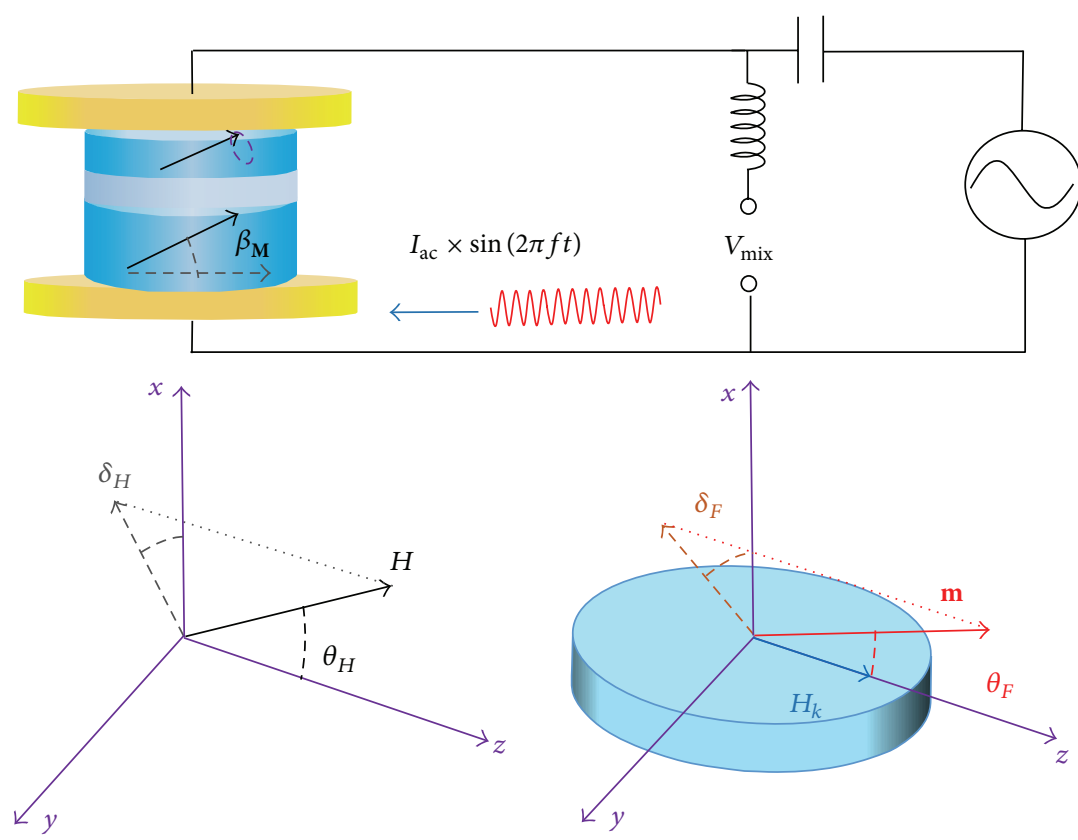

(a)

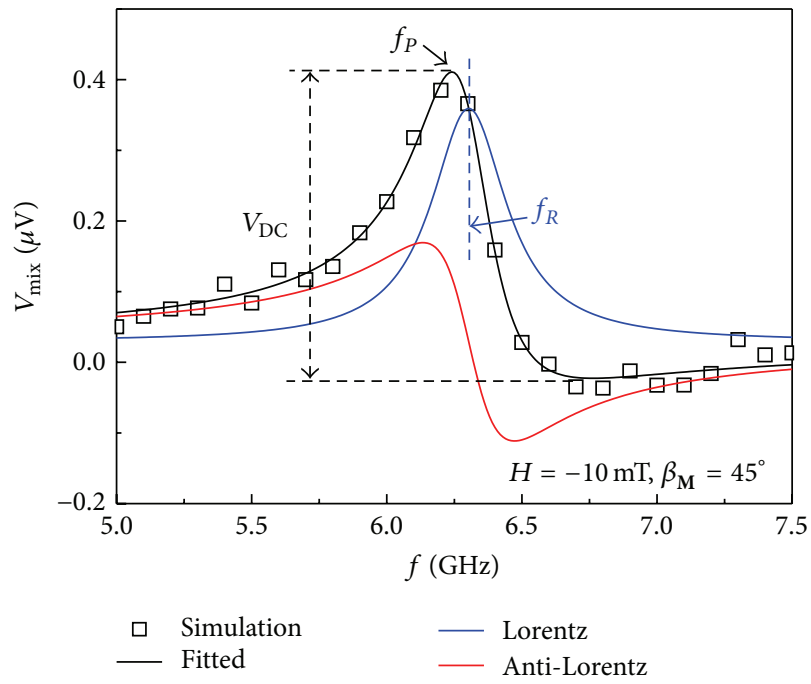

(b)

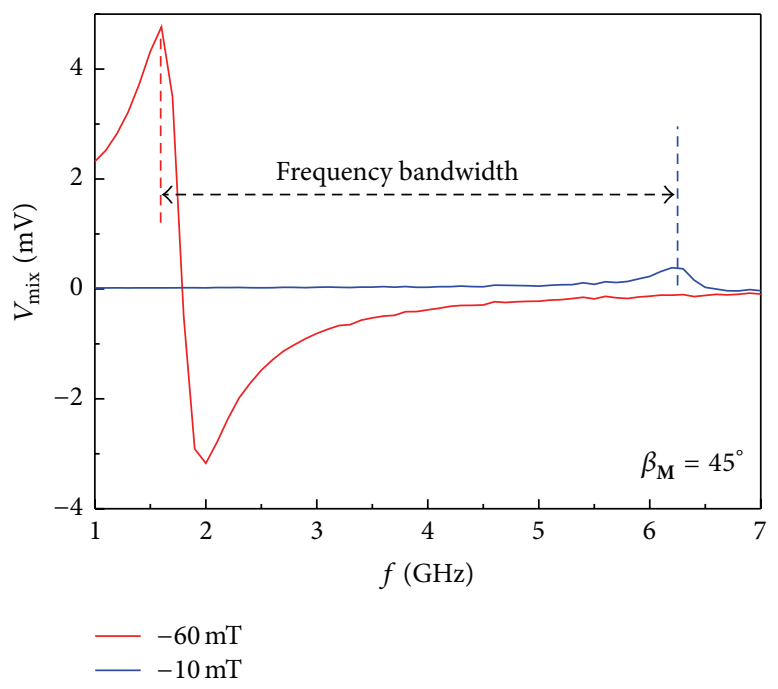

(c)

Figure 1: (a) AC is injected into an STMD under magnetic field $(H)$ applied at $\left(\theta_{H}, \delta_{H}\right)$, and the free-layer magnetization $\left(\theta_{F}, \delta_{F}\right)$ precesses around its equilibrium; (b) a typical $V_{\text {mix }}-f$ spectrum can be decomposed into a Lorentz peak and an anti-Lorentz curve; (c) frequency bandwidth is defined as frequency gap of $f_{P}$ at $\mu_{0} H=-10 \mathrm{mT}$ and $-60 \mathrm{mT}$.

exceed that of the existing Schottky diode detector, the effects of these approaches on bandwidth and NEP are seldom mentioned. On the other hand, the number of reports aiming at extending the bandwidth or reducing the NEP is small. It has been shown that the bandwidth can be extended through the introduction of reference layer with tilted magnetization [17]. It has also been shown that the minimum detection power of an STMD can be reduced through working at cryogenic temperatures [18]. A comprehensive investigation on how these three performance indicators are influenced by the material or operational parameters is required to extend the understanding in the behavior of STMDs. The outcome of this work is beneficial for optimizing the performance of STMDs.

\section{Modeling and Computational Details}

The device under investigation is a $200 \times 100 \mathrm{~nm}^{2}$ elliptical MTJ nanopillar with reference-layer magnetization (M) tilted out of plane by $\beta_{\mathrm{M}}$ (Figure $\left.1(\mathrm{a})\right) . H$ is applied at $3 \mathrm{D}$ direction defined by the polar $\left(\theta_{H}\right)$ and azimuthal $\left(\delta_{H}\right)$ angles. In 
a coordinate system where the $x$-axis is perpendicular to the thin-film plane and the $z$-axis is parallel to the magnetic easy axis, the unit vector of $\mathbf{M}$ and free-layer magnetization ( $\mathbf{m}$ ) can be written as

$$
\begin{aligned}
\vec{M} & =\left(\cos \beta_{\mathbf{M}}, 0, \sin \beta_{\mathbf{M}}\right), \\
\vec{m} & =\left(\sin \theta_{F} \cos \delta_{F}, \sin \theta_{F} \sin \delta_{F}, \cos \theta_{F}\right) .
\end{aligned}
$$

Microwave current $I(t)=I_{\mathrm{ac}} \sin (2 \pi f t)$ with $I_{\mathrm{ac}}=10 \mu \mathrm{A}$ and $f=0.1-10 \mathrm{GHz}$ is injected into the MTJ and a steady-state oscillation in $\mathbf{m}$ is excited by the STT of spin current. Time evolution of $\mathbf{m}\left(\theta_{F}(t), \delta_{F}(t)\right)$ can be obtained by numerically solving the Landau-Lifshitz-Gilbert equation:

$$
\begin{aligned}
\frac{d \vec{m}}{d t} & =-\gamma \vec{m} \times\left[\vec{H}_{\mathrm{eff}}+\vec{H}_{\text {therm }}(t)\right]+\alpha \vec{m} \times \frac{d \vec{m}}{d t}-\gamma \\
& \cdot \frac{I(t) h}{4 \pi e \mu_{0} M_{s} V} \\
& \cdot \frac{P}{1+P^{2} \cos (\varphi(t))}\left[\vec{m} \times(\vec{m} \times \vec{M})+b_{f} \vec{m} \times \vec{M}\right],
\end{aligned}
$$

where $\gamma=176 \mathrm{GHz} / \mathrm{T}$ represents the gyromagnetic ratio, $\alpha=$ 0.01 the Gilbert damping parameter, $V$ the volume of free layer, $e$ the charge of an electron, and $\mu_{0}$ the vacuum magnetic permeability. $H_{\text {eff }}$ is the effective field comprised of external field, IP anisotropy field $\left(H_{k}\right)$, and demagnetization field $\left(H_{d}\right)$, defined, respectively, by

$$
\begin{aligned}
& H_{k}=N_{x} \mu_{0} M_{S} \\
& H_{d} \approx \mu_{0} M_{S},
\end{aligned}
$$

where $N_{x}=0.016$ is the demagnetization factor in the $x$-axis. $\varphi(t)$ is the time-dependent angle between $\mathbf{M}$ and $\mathbf{m}$ :

$$
\begin{aligned}
\cos \varphi(t)= & \vec{m} \cdot \vec{M} \\
= & \sin \theta_{F}(t) \cdot \cos \delta_{F}(t) \cdot \cos \beta_{\mathbf{M}}+\cos \theta_{F}(t) \\
& \cdot \sin \beta_{\mathbf{M}} .
\end{aligned}
$$

The contribution of field-like STT is considered, and $b_{f}=0.1$ is its ratio to the IP torque [19]. The thermal fluctuation term $\left(\vec{H}_{\text {therm }}(t)\right)$ is introduced as in [20]:

$$
\vec{H}_{\text {therm }}(t)=\vec{\xi}(t) \sqrt{\frac{\alpha}{1+\alpha^{2}} \frac{2 k_{B} T}{\gamma \mu_{0} M_{S} V}},
$$

where $\vec{\xi}(t)$ is a random vector whose components are normally distributed random numbers with mean of 0 and variance of 1 . The temperature dependence of polarization ratio $(P)$, parallel resistance $\left(R_{P}\right)$, antiparallel resistance $\left(R_{\mathrm{AP}}\right)$, and saturation magnetization $\left(M_{S}\right)$ are expressed, respectively, as

$$
\begin{aligned}
P(T) & =P(0) \times\left(1-\eta T^{3 / 2}\right), \\
R_{P}(T) & =\frac{R(0) \times(1-\chi T)}{1+P(T)^{2}},
\end{aligned}
$$

$$
\begin{aligned}
& R_{\mathrm{AP}}(T)=\frac{R(0) \times(1-\chi T)}{1-P(T)^{2}}, \\
& M_{S}(T)=M_{S}(0) \times\left(1-\frac{T}{T_{C}}\right)^{\tau},
\end{aligned}
$$

where $P(0)=0.385, \eta=1.7 \times 10^{-5} \mathrm{~K}^{-3 / 2}, R(0)=800 \Omega, \chi=7.65$ $\times 10^{-4} \mathrm{~K}^{-1}, M_{S}(0)=1.3 \times 10^{6} \mathrm{~A} / \mathrm{m}, \tau=0.4$, and $T_{C}=1300 \mathrm{~K}$.

The oscillation of $\mathbf{m}$ results in the changing resistance of the MTJ:

$$
R(t)=\left[\frac{R_{\mathrm{AP}}^{-1}+R_{P}^{-1}}{2}-\frac{R_{P}^{-1}-R_{\mathrm{AP}}^{-1}}{2} \cos \varphi(t)\right]^{-1} .
$$

The changing resistance is thus mixed with the alternating current, generating a mixing voltage $\left(V_{\text {mix }}\right)$ on the MTJ defined as the average voltage over one period:

$$
V_{\text {mix }}=\frac{\int_{t_{0}}^{t_{0}+1 / f} I(t) \cdot R(t) d t}{1 / f} .
$$

A typical $V_{\text {mix }}-f$ spectrum can be decomposed into a symmetric Lorentz peak, contributed by the IP STT, and an anti-Lorentz curve resulted from the field-like torque [21] (Figure 1(b)):

$$
\begin{gathered}
V_{\text {mix }}=A I_{R F}^{2}+B I_{R F}^{2}\left[\frac{1}{1+\left(\left(f-f_{R}\right) / \sigma\right)^{2}}\right. \\
\left.-C \frac{f-f_{R}}{\sigma} \frac{1}{1+\left(\left(f-f_{R}\right) / \sigma\right)^{2}}\right],
\end{gathered}
$$

where $f_{R}$ and $\sigma$ are the frequency and line-width of the ferromagnetic resonant (FMR) peak, respectively. $A$ and $B$ are constants related to the nonlinearity of junction resistance, while $C$ is related to the content of field-like STT. This combined contribution has resulted in a peak and a valley at either side of $f_{R}$ in the $V_{\text {mix }}-f$ spectrum. If the IP STT is dominating, more symmetric curve will be observed and $f_{P}$ approaches $f_{R}$. On the other hand, if the proportion of field-like STT is higher, the curve will be more dispersed and the resonant peak shifts away from $f_{R}$. The output DC voltage $\left(V_{\mathrm{DC}}\right)$ is calculated as the voltage difference between the peak and valley in the $V_{\text {mix }}-f$ spectrum. Considering the microwave reflection caused by impedance mismatch, the sensitivity and NEP [15] can be calculated from

$$
\begin{aligned}
& \text { Sensitivity }=\frac{V_{\mathrm{DC}}}{(1 / 8)\left(\left(\left(R+Z_{0}\right)^{2} \cdot I_{\mathrm{ac}}^{2}\right) / Z_{0}\right)}, \\
& \mathrm{NEP}=\frac{\text { noise }}{\text { sensitivity }} \\
& \quad=\sqrt{8} \pi \frac{\alpha}{P(T)^{2}} \frac{M_{S}(T)}{\mu_{B}} V \frac{Z_{0}}{h / e^{2}}\left(\frac{R+Z_{0}}{Z_{0}}\right)^{2} k_{B} T \sqrt{\sigma},
\end{aligned}
$$

where $R$ is the average junction resistance and $Z_{0}=50 \Omega$ is the characteristic impedance of the transmission lines. 
In this work, one material parameter $\left(\beta_{\mathbf{M}}\right)$ and two operational parameters (the orientation of magnetic field and the temperature) are selected as the influencing parameters for optimizing detector performance. Firstly, the reliance of $f_{R}$, sensitivity, and NEP on $H$ is analyzed to explore the mechanism for the performance tuning. Later on, the suitable material for reference layer is suggested based on optimal $\beta_{\mathbf{M}}$ for highest sensitivity. The influence of operational parameters (the orientation of magnetic field and temperature) is subsequently studied. Finally, the approaches to achieve optimized performance in STMDs are discussed.

\section{Simulation Results}

3.1. Magnitude of Magnetic Field $(H)$. Since $f_{P}$ and $V_{\mathrm{DC}}$ of STMDs are primarily influenced by $H$, the voltage response under different $H$ is firstly studied to explore the mechanism for the changes in $f_{P}$, sensitivity, and NEP. In these simulations, $\mathbf{M}$ is tilted OOP $\left(\beta_{\mathbf{M}}=40^{\circ}\right)$ while the magnetic field is applied in the hard plane $\left(\delta_{H}=40^{\circ}\right.$ ) (Figure 2(a)). The contour plots of $V_{\text {mix }}-f$ spectra when $\mu_{0} H$ is changed from $-10 \mathrm{mT}$ to $-150 \mathrm{mT}$ are shown in Figure 2(b). $f_{R}$ and $f_{P}$ are shown in Figure 2(c). $f_{R}$ tends to decrease with $H$ when $\mu_{0} H<-64 \mathrm{mT}$ but increase with $H$ thereafter. This $H$ dependence of FMR frequency [22, 23] and line-width [24] can be explained by the following model:

$$
\begin{aligned}
f_{R} & =\frac{\gamma}{2 \pi} \sqrt{H_{\mathrm{eff}} B_{m}}=\frac{\gamma}{2 \pi} \sqrt{H_{\mathrm{eff}}\left(H_{\mathrm{eff}}+\mu_{0} M_{S}\right)}, \\
\sigma & \approx-\alpha \gamma\left(2\left|H_{\mathrm{eff}}\right|+H_{c}\right)
\end{aligned}
$$

where $H_{\text {eff }}$ is the effective field represented by

$$
H_{\text {eff }}=H-H_{\text {an }}
$$

where $H_{\text {an }}$ represents the anisotropy field. The $\mathrm{V}$-shape relation between $f_{R}$ and $H$ resulted from the competition between the external magnetic field and the anisotropy field. When $\mu_{0} H=-64 \mathrm{mT}$, the free-layer anisotropy is overcome by the external magnetic field, resulting in minimum $H_{\text {eff }}$ and thus minimum $f_{R}$, according to (16). At larger $H, H_{\text {eff }}$ increases with $H$, resulting in increasing $f_{R}$. The field dependence of $f_{P}$ is nearly the same as that of $f_{R}$. This constant frequency gap between $f_{P}$ and $f_{R}$ is contributed by the constant ratio between IP and field-like STT. Since the range of $f_{P}$ is constrained by the range of $H$, the frequency bandwidth mentioned in the following is calculated as the bandwidth of $f_{P}$ when the $\mu_{0} H$ is changed from $-10 \mathrm{mT}$ to $-60 \mathrm{mT}$. For example, a bandwidth of $4.6 \mathrm{GHz}$ is achieved in this situation $\left(f_{R}\right.$ decreasing monotonously from $6.2 \mathrm{GHz}$ at $-10 \mathrm{mT}$ to $1.6 \mathrm{GHz}$ at $-60 \mathrm{mT}) . \sigma$ shares similar $H$ dependence with $f_{R}$ (Figure $2(\mathrm{~d})$ ) since it is proportional to the magnitude of $H_{\text {eff }}$, according to (17). It should be noted that highest sensitivity, $127 \mathrm{mV} / \mathrm{mW}$, is achieved with the minimum frequency (Figure 2(e)). This can be explained by an analytical model proposed by Wang et al. [10]. The $V_{\mathrm{DC}}$ of an STMDs can be expressed as

$$
\begin{aligned}
V_{\mathrm{DC}}= & \frac{R_{\mathrm{AP}}-R_{P}}{R_{P}} \frac{\mu_{B}}{2 e\left(M_{S} A\right) \sigma} \frac{P}{1+P^{2}} \frac{R^{2}}{R_{P} R_{\mathrm{AP}}} \frac{I_{\mathrm{ac}}^{2}}{8} R \\
& \cdot \sin ^{2} \varphi .
\end{aligned}
$$

When $\mu_{0} H=-64 \mathrm{mT}$, the free-layer magnetization is saturated in the direction of the hard-plane magnetic field [25], so the angle $(\varphi)$ between $\mathbf{m}$ and $\mathbf{M}$ reaches $90^{\circ}$. This results in maximum $R$ according to (11). As $\sigma$ also reaches the minimum, the $V_{\mathrm{DC}}$ and the sensitivity are thus expected to arrive at the maximum. Meanwhile, the calculated NEP in Figure 2(f) presents similar reliance on $H$ as the line-width. This is because NEP is proportional to the square root of $\sigma$ as inferred from (15). In the following investigation, $\mu_{0} H=$ $-60 \mathrm{mT}$ is used since it is beneficial for high sensitivity and low NEP. The above analysis has revealed that the mechanism in the tuning of $f_{R}, \sigma$, sensitivity, and NEP can all be explained through analyzing the changes in $H_{\text {eff }}$ and $\varphi$. Similarly, it can be inferred that through tuning the material and operational parameters of STMDs, changes in $H_{\text {eff }}$ and $\varphi$ can also be induced. The altered performance indicators shown below can also be analyzed with the same model.

3.2. Tilt Angle of Reference Layer. OOP anisotropy is reported in $\mathrm{Co} / \mathrm{Pt}$ [26] or $\mathrm{Co} / \mathrm{Ni}$ [27] multilayers and annealed single layers [28]. $\beta_{\mathbf{M}}$ can be carefully tuned through adopting reference-layer materials with different OOP anisotropy. The angular dependence of sensitivity, bandwidth, and NEP is analyzed to explore an optimal $\beta_{\mathbf{M}}$. The direction of magnetic field is applied at $\theta_{H}=90^{\circ}$ and $\delta_{H}=40^{\circ}$ while $\beta_{\mathbf{M}}$ is changed from $0^{\circ}$ to $180^{\circ}$. The contour plots of $V_{\text {mix }}-f$ spectra simulated at different $\beta_{\mathrm{M}}$ are shown in Figure 3(a). Observed $f_{R}$ and $\sigma$ present only slight changes at various $\beta_{\mathrm{M}}$ (Figures $3(\mathrm{~b})$ and $3(\mathrm{c})$ ). This indicates that the STMD works at free-layer resonation mode, so the resonant frequency is immune to the changes in the reference layer. Since the magnetic field is applied in the $x-y$ plane, the $f_{R}$ and $\sigma$ are roughly symmetric when the IP component of $\mathbf{M}$ is parallel $\left(\beta_{\mathbf{M}}<90^{\circ}\right)$ and antiparallel $\left(\beta_{\mathbf{M}}>90^{\circ}\right)$ to the $z$ direction. However, angular dependent and asymmetric $f_{P}$ is observed in Figure $3(\mathrm{~b})$. The larger frequency difference between $f_{R}$ and $f_{P}$ when $\mathbf{M}$ is tilted by $10^{\circ}-80^{\circ}$ or $10^{\circ}-170^{\circ}$ indicates that the $V_{\text {mix }}-f$ relationship is dominated by the field-like STT. The asymmetry is due to the different contribution of STT (positive when $\beta_{\mathrm{M}}<90^{\circ}$, negative when $\beta_{\mathbf{M}}>90^{\circ}$ ). The resulting frequency bandwidth reaches maximum when $\beta_{\mathbf{M}}=75^{\circ}$ (Figure $3(\mathrm{~d})$ ). This is consistent with our previous simulation results that the bandwidth can be extended by optimizing $\beta_{M}$ [17]. However, since very narrow $H$ range $(50 \mathrm{mT})$ is used in this work, the changes in the bandwidth are also relatively small. Apart from bandwidth, the sensitivity and NEP are also angular dependent. The sensitivity reaches maximum when $\beta_{\mathbf{M}}=\sim 45^{\circ}$ and $\sim 135^{\circ}$ (Figure 3(e)). This is because largest $\varphi$ at these angles results in large $V_{\mathrm{DC}}$ according to (19). The calculated NEP presents a trend to slightly decrease at larger $\beta_{M}$ in Figure 3(f). Considering that $\sigma$ is nearly constant, the low NEP is contributed by 


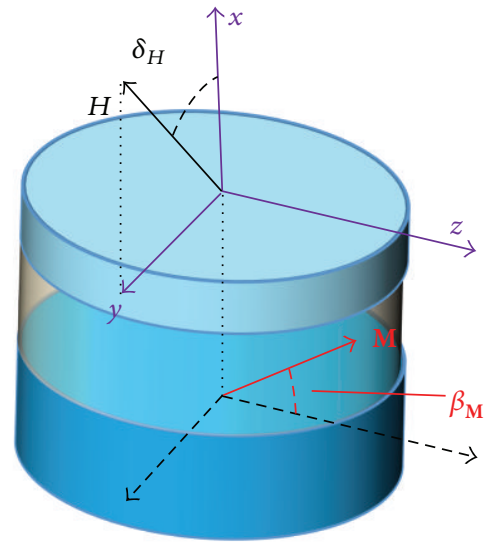

(a)

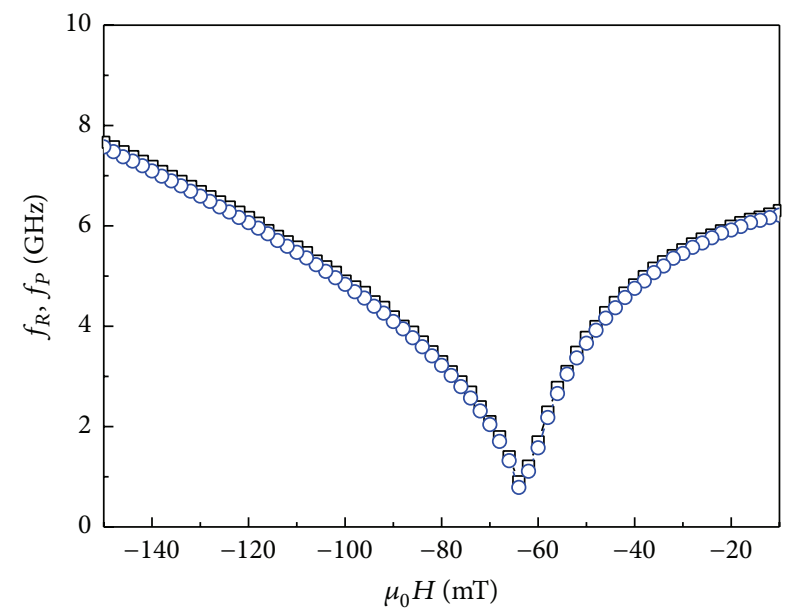

$\rightarrow-f_{R}$
$-0-f_{P}$

(c)

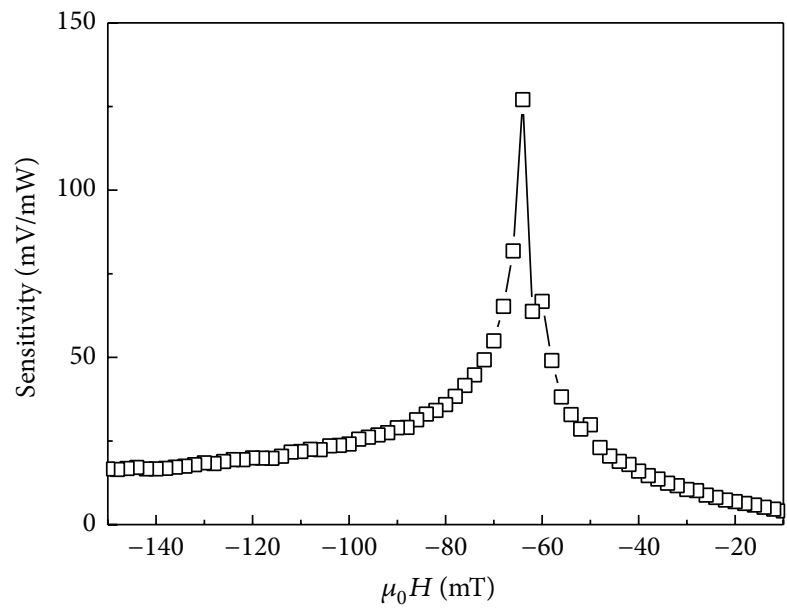

(e)

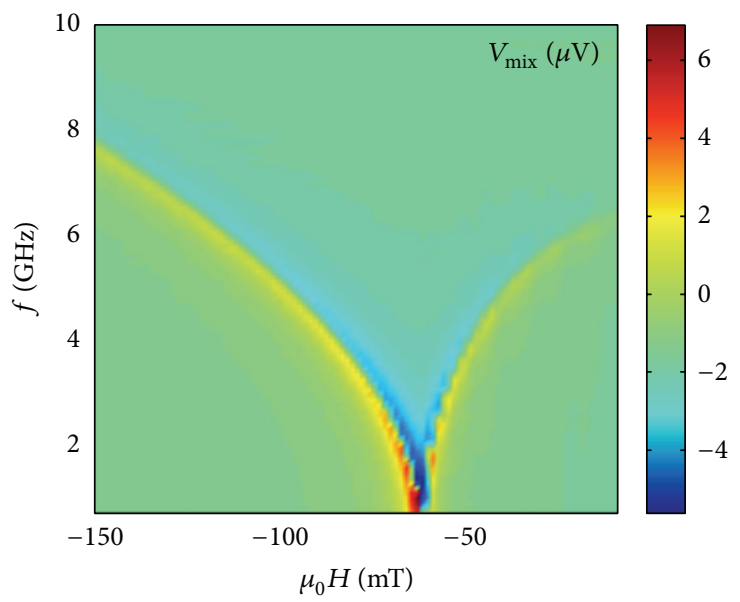

(b)

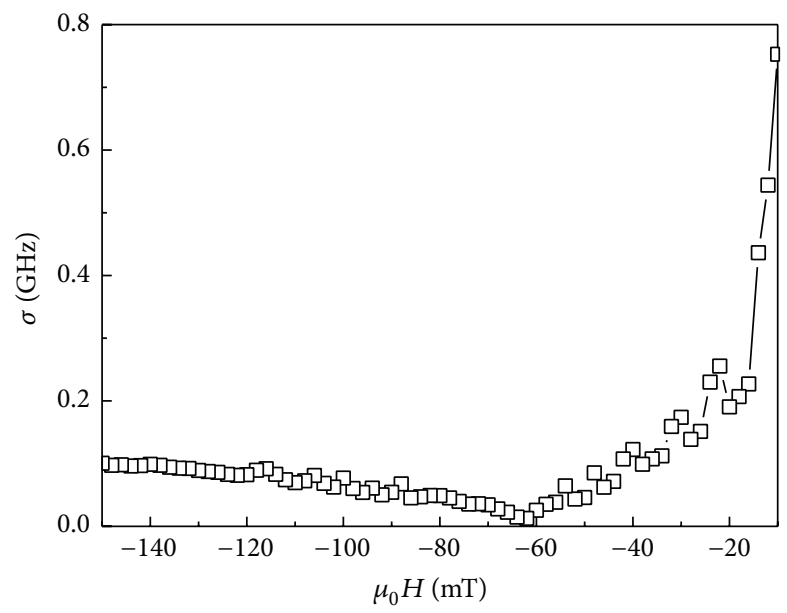

(d)

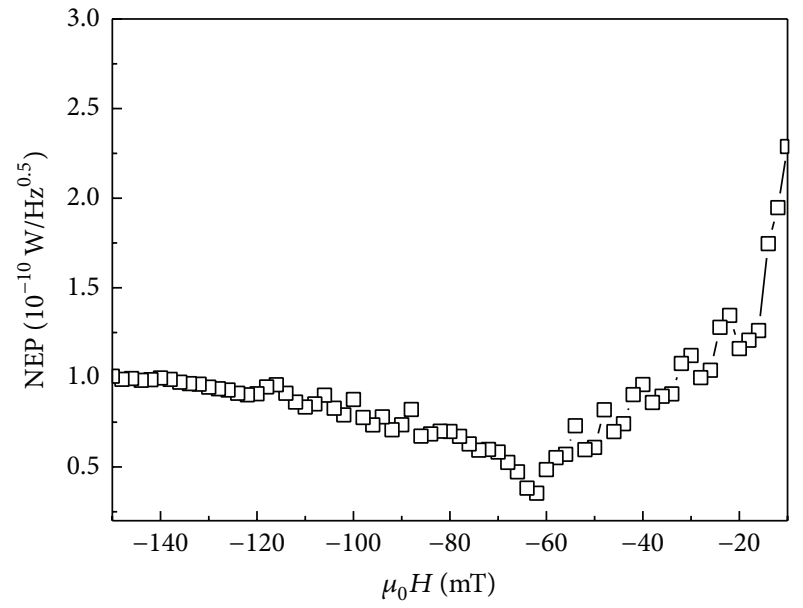

(f)

Figure 2: (a) Schematic of alignment between $H$ and M, (b) $V_{\text {mix }}-f$ spectra, (c) FMR frequency $\left(f_{R}\right)$ and peak frequency $\left(f_{P}\right)$, (d) line-width, (e) sensitivity, and (f) NEP when the magnitude of magnetic field is changed from $-10 \mathrm{mT}$ to $-150 \mathrm{mT}$. 

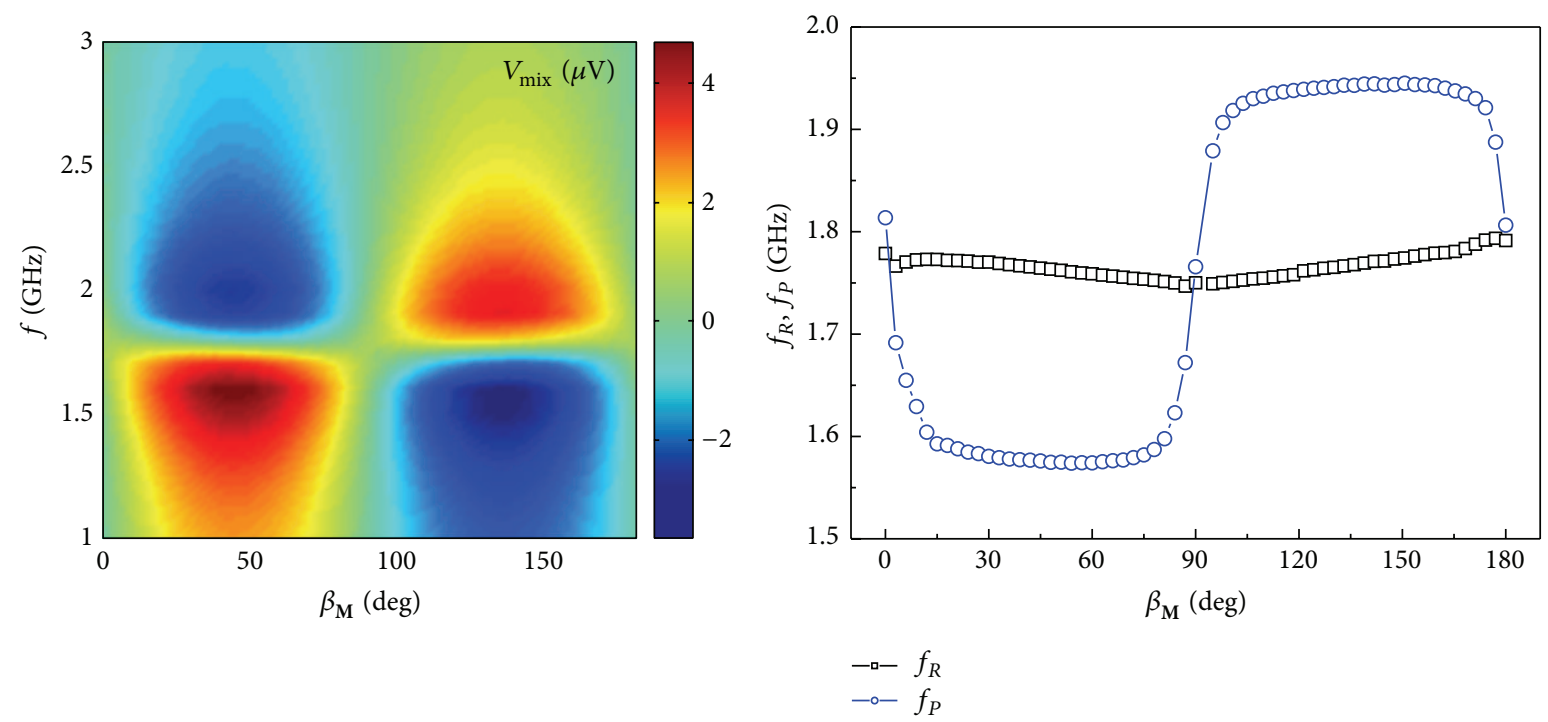

(a)

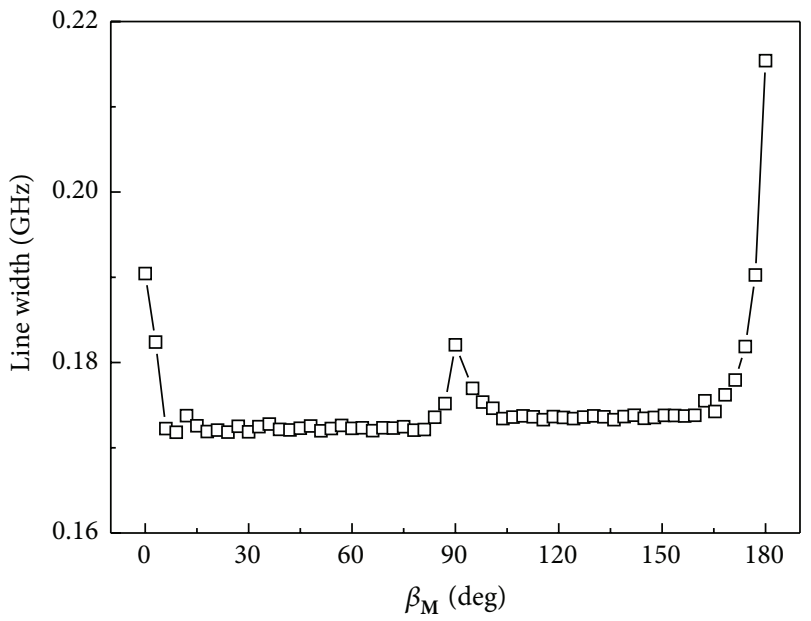

(b)

(c)

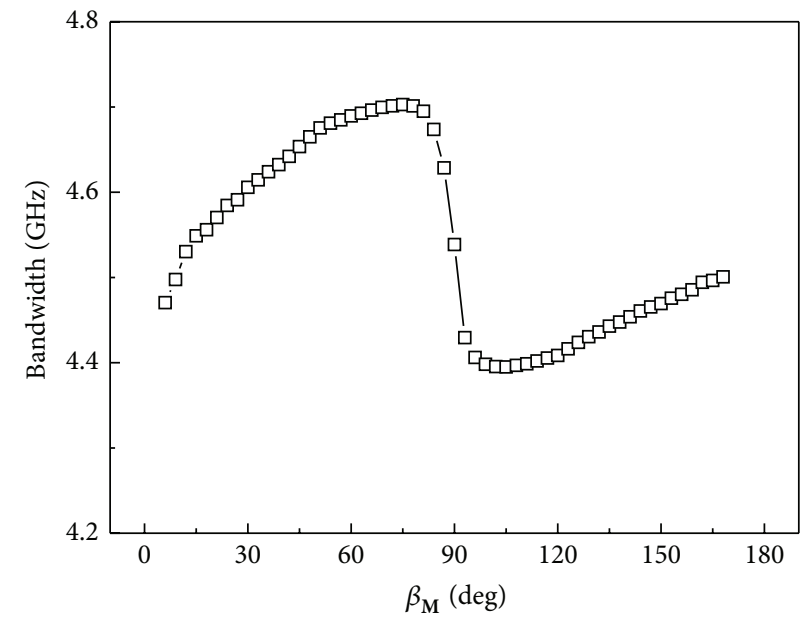

(d)

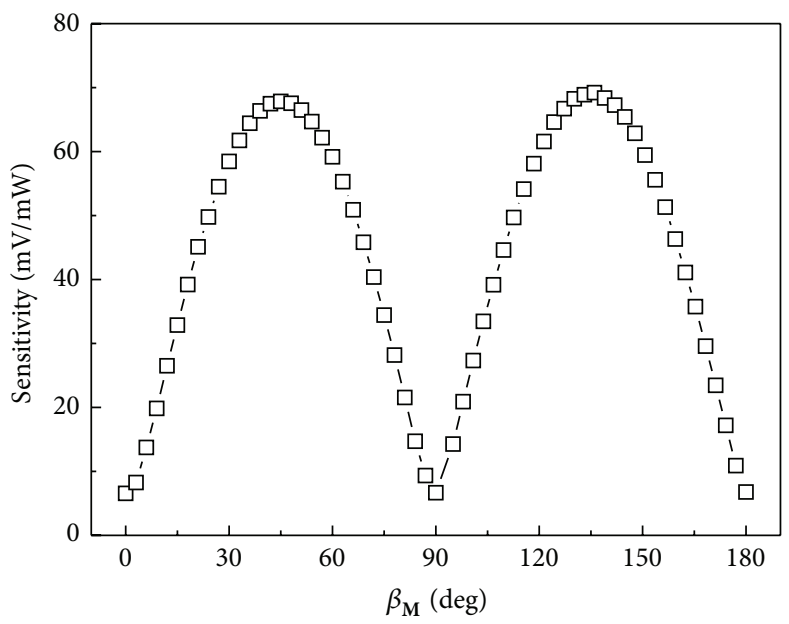

(e)

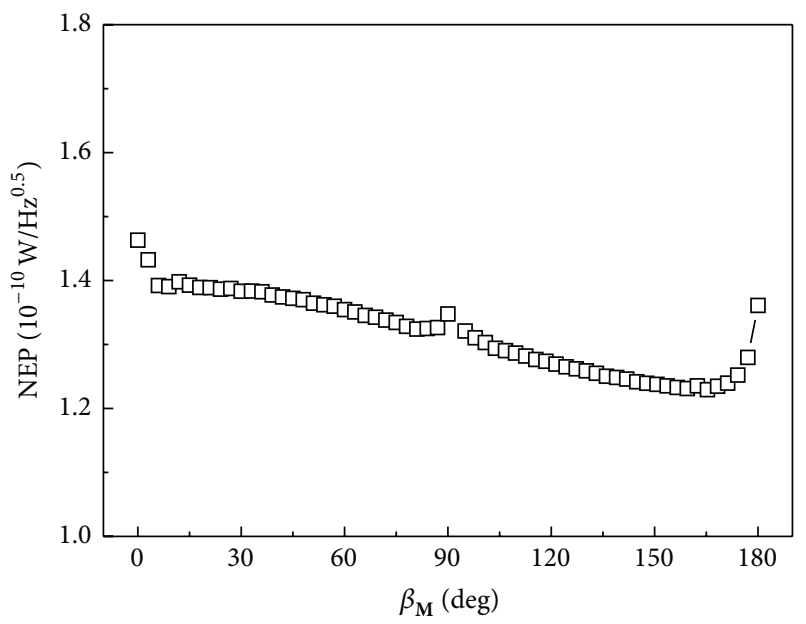

(f)

Figure 3: (a) The contour plots of $V_{\text {mix }}-f$ spectra, (b) $f_{R}$ and $f_{P}$, (c) line-width, (d) bandwidth, (e) sensitivity, and (f) NEP as a function of $\beta_{\mathrm{M}}$ when $\mu_{0} H=-60 \mathrm{mT}$ is applied at $\theta_{H}=90^{\circ}$ and $\delta_{H}=40^{\circ}$. 
the relatively lower junction resistance at larger $\beta_{\mathrm{M}}$. From the above analysis, we can infer that optimal $\beta_{\mathrm{M}}$ is $45^{\circ}$ or $135^{\circ}$, where highest sensitivity can be achieved, while the bandwidth and NEP are only slightly influenced. In the following discussion, $\beta_{\mathbf{M}}=45^{\circ}$ is used. Materials such as $\mathrm{L}_{0}(101) \mathrm{FePt}$ is suggested as the reference layer to achieve $\beta_{M}=45^{\circ}[29,30]$.

3.3. Orientation of OOP Magnetic Field. The direction of magnetic field can be changed to achieve the preferred performance in STMDs. In this investigation, the direction of $-60 \mathrm{mT}$ magnetic field is changed within the range of $\theta_{H}=$ $0^{\circ}-90^{\circ}$ and $\delta_{H}=10^{\circ}-90^{\circ}$ (Figure $4(\mathrm{a})$ ). Higher $f_{R}$ is observed at smaller $\theta_{H}$ (Figure $4(\mathrm{~b})$ ), due to the higher effective field when the magnetic field is applied IP. The angular dependence of bandwidth in Figure 4(c) is roughly consistent with that of $f_{R}$ at $\mu_{0} H=-60 \mathrm{mT}$ because, in most cases, higher $f_{P}$ and $f_{R}$ occur at larger $H$. However, when $\theta_{H}$ approaches $90^{\circ}$ and $\delta_{H}$ is small, very narrow bandwidth is observed. This is consistent with our experimental observations that when the magnetic field is nearly perpendicular to plane, $f_{P^{-}}$ tunability by $H$ is much reduced (not shown in this paper). Similar to Figure 2, the narrow line-width (Figure 4(d)) coexists with low $f_{R}$ because they have similar reliance on $H_{\text {eff }}$ from (16) and (18). Small $\sigma$ at larger $\theta_{H}$ also results in the highest sensitivity (Figure 4(e)) and smallest NEP (Figure 4(f)) when $\theta_{H}=90^{\circ}$ and $\delta_{H}=40^{\circ}$. These results have indicated a contradiction between high sensitivity and wide bandwidth when choosing the optimal field angle: larger $\theta_{H}$ is beneficial for high sensitivity and low NEP, but it also results in a reduction in bandwidth; widest bandwidth over $10 \mathrm{GHz}$ can be achieved when $\theta_{H}=0^{\circ}$, while it comes with a drawback of low sensitivity below $10 \mathrm{mV} / \mathrm{mW}$ and high NEP over $3 \times$ $10^{-10} \mathrm{~W} / \mathrm{Hz}^{0.5}$. Nevertheless, these results have shown that the performance of STMDs can be tailored in a wide range by changing the orientation of magnetic field.

3.4. Working Temperature. The above investigations have shown that the highest sensitivity can be achieved when the reference-layer magnetization is tilted by $\beta_{\mathrm{M}}=45^{\circ}$ and the magnetic field is applied at $\theta_{H}=90^{\circ}$ and $\delta_{H}=40^{\circ}$. Based on this optimized alignment, the working temperature is further studied as another influencing parameter to tune the STMD performance. The contour plots of $V_{\text {mix }}-f$ spectra when temperature changes from $5 \mathrm{~K}$ to $380 \mathrm{~K}$ are shown in Figure 5(a). Calculated $f_{R}$ increases monotonously with decreasing temperature (Figure 5(b)), which resulted from increasing $H_{\text {eff }}$ contributed by higher $M_{S}$ at low temperature. $f_{P}$ at $\mu_{0} H=$ $-60 \mathrm{mT}$ also changes from $3.4 \mathrm{GHz}$ to $0.4 \mathrm{GHz}$ as temperature increases from $5 \mathrm{~K}$ to $380 \mathrm{~K}$. In contrast, $f_{P}$ at $\mu_{0} H$ $=-10 \mathrm{mT}$ presents much smaller temperature dependence, which decreases from $7 \mathrm{GHz}$ to $6.1 \mathrm{GHz}$ within the same temperature range. As a result, the frequency bandwidth shown in Figure 5(c) increases with temperature from $3.6 \mathrm{GHz}$ to $5.7 \mathrm{GHz}$. Decreasing $f_{R}$ at high temperature results in narrower line-width shown in Figure 5(d). It is noted that the calculated sensitivity increases with temperature (Figure 5(e)), which contradicts with the previous simulation report [18]. The reasons for this temperature dependence can be interpreted as follows. Firstly, $M_{S}, P$, and $\sigma$ increase at lower temperature. Secondly, equilibrium $\varphi$ under $\mu_{0} H=-60 \mathrm{mT}$ is reduced at lower temperature due to the higher anisotropy. Thirdly, the higher resistance at low temperature results in higher microwave reflection coefficient, leading to smaller $I_{\mathrm{ac}}$ when constant microwave power is applied. All these reduction effects at lower temperature have overwhelmed the increasing effect brought by higher TMR ratio, so a reduction in $V_{\mathrm{DC}}$ and sensitivity is expected with decreasing temperature according to (19). The calculated NEP increases with temperature, as shown in Figure 5(f). This is reasonable since the level of noise is expected to increase at higher temperature. As such, increasing the working temperature is beneficial for increasing the sensitivity and frequency bandwidth. However, a drawback of increased NEP is accompanied.

\section{Discussion}

Based on the above analysis, the approaches for optimizing the performance of STMDs can be inferred. The sensitivity can be optimized while maintaining bandwidth and NEP nearly unchanged by adopting a reference layer tilted at $\beta_{M}=$ $45^{\circ}$. However, the attempt to further improve the sensitivity through changing the direction of magnetic field or working temperature is accompanied by a reduction in bandwidth or an increase in NEP. Fortunately, through the combined manipulation of the two operational parameters, a balance among the sensitivity, bandwidth, and NEP can be achieved based on the specific applications. In a situation where high sensitivity is needed, the magnetic field can be applied at $\theta_{H}=$ $90^{\circ}$ and $\delta_{H}=40^{\circ}$ to achieve the peak sensitivity. The resulting reduction in bandwidth can be partially compensated by increasing the working temperature, which also contributes to higher sensitivity. Meanwhile, the resulting high NEP is also reduced at the optimized orientation of magnetic field. Similarly, when a wide-band STMD is preferred, small $\theta_{H}$ can be used to achieve wide bandwidth, and the loss of sensitivity can be made up by increasing temperature. These results reveal a new degree of freedom in tailoring the performance of STMDs. Through altering the operational parameters, the regime of the applications of STMDs is remarkably extended.

\section{Conclusions}

In summary, the influencing parameters on the performance of STMDs are evaluated through macrospin simulation. The alignment between $H$ and $\mathbf{M}$ is optimized and the temperature dependence of sensitivity, bandwidth, and NEP are investigated. The $\mathrm{V}$-shape reliance of $f_{R}$ and $\sigma$ on $H$ is interpreted through evaluating $H_{\text {eff }}$ and $\varphi$. When $\mathbf{M}$ is tilted OOP by $\beta_{\mathrm{M}}=45^{\circ}$, highest sensitivity can be achieved, while the bandwidth and line-width exhibit small angular dependence. Higher sensitivity and lower NEP are observed when the direction of magnetic field approaches the hard plane $\left(\theta_{H}=90^{\circ}\right)$ due to larger $\varphi$ and smaller $\sigma$. However, the high sensitivity is accompanied by a reduction in bandwidth. Higher working temperature results in higher sensitivity and wide bandwidth at the cost of increased NEP. Although the accomplishment of optimized sensitivity, bandwidth, and NEP by one single approach is not yet possible, these results 


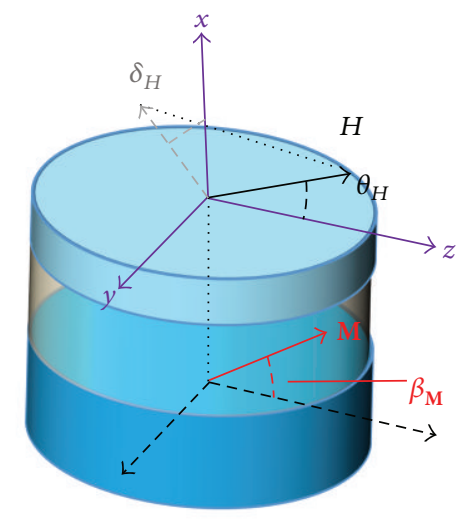

(a)

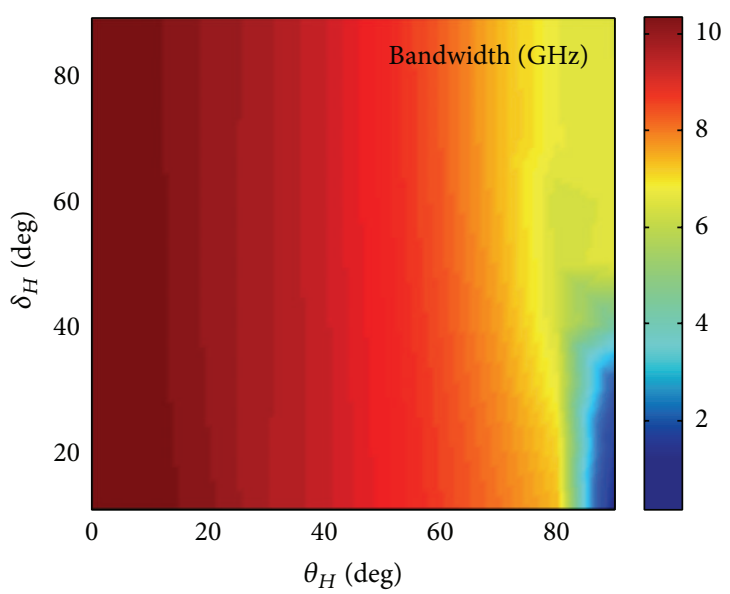

(c)

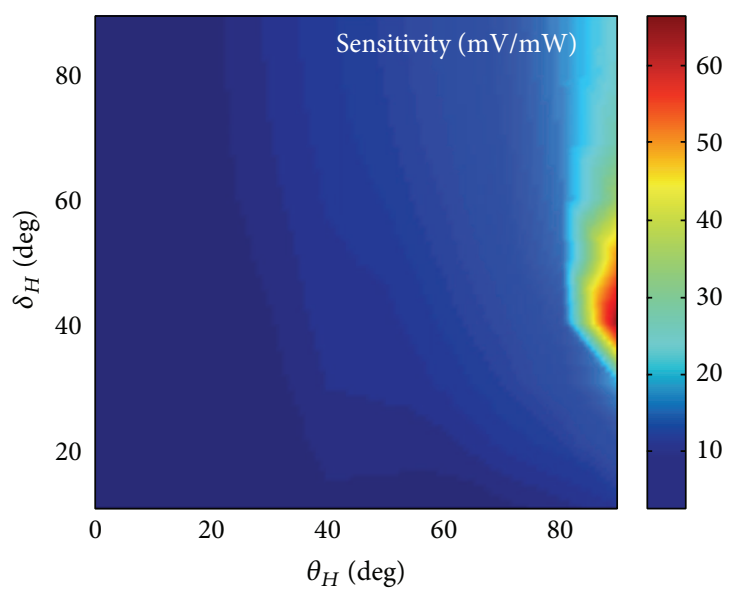

(e)

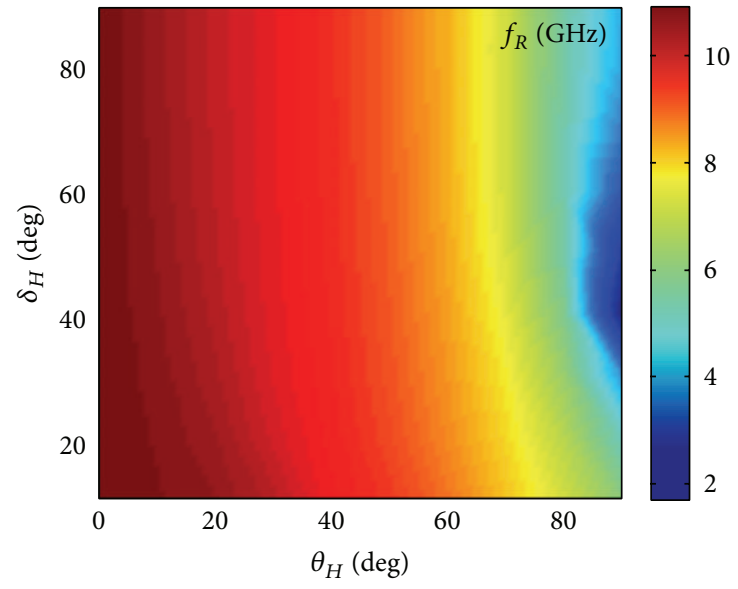

(b)

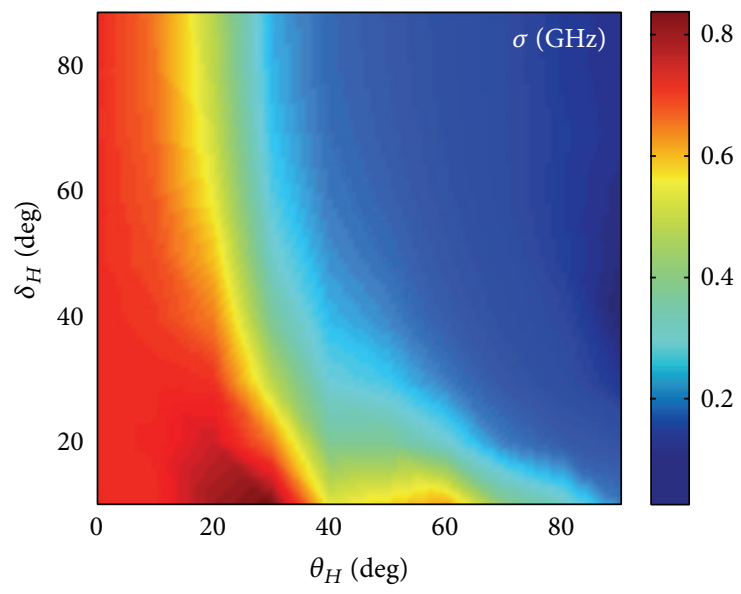

(d)

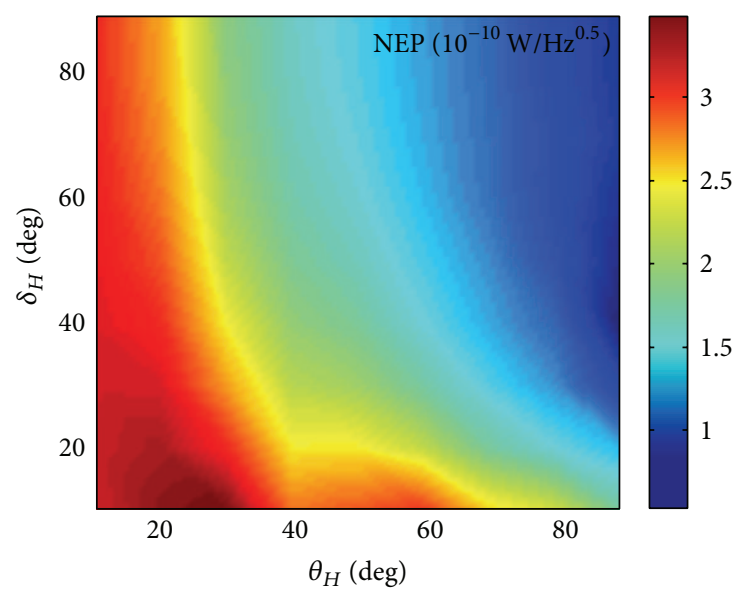

(f)

FIgure 4: (a) Schematic of OOP magnetic field, (b) $f_{R}$, (c) bandwidth, (d) line-width, (e) sensitivity, and (f) NEP as a function of orientation angles of magnetic field $\left(\mu_{0} H=-60 \mathrm{mT}\right)$. 

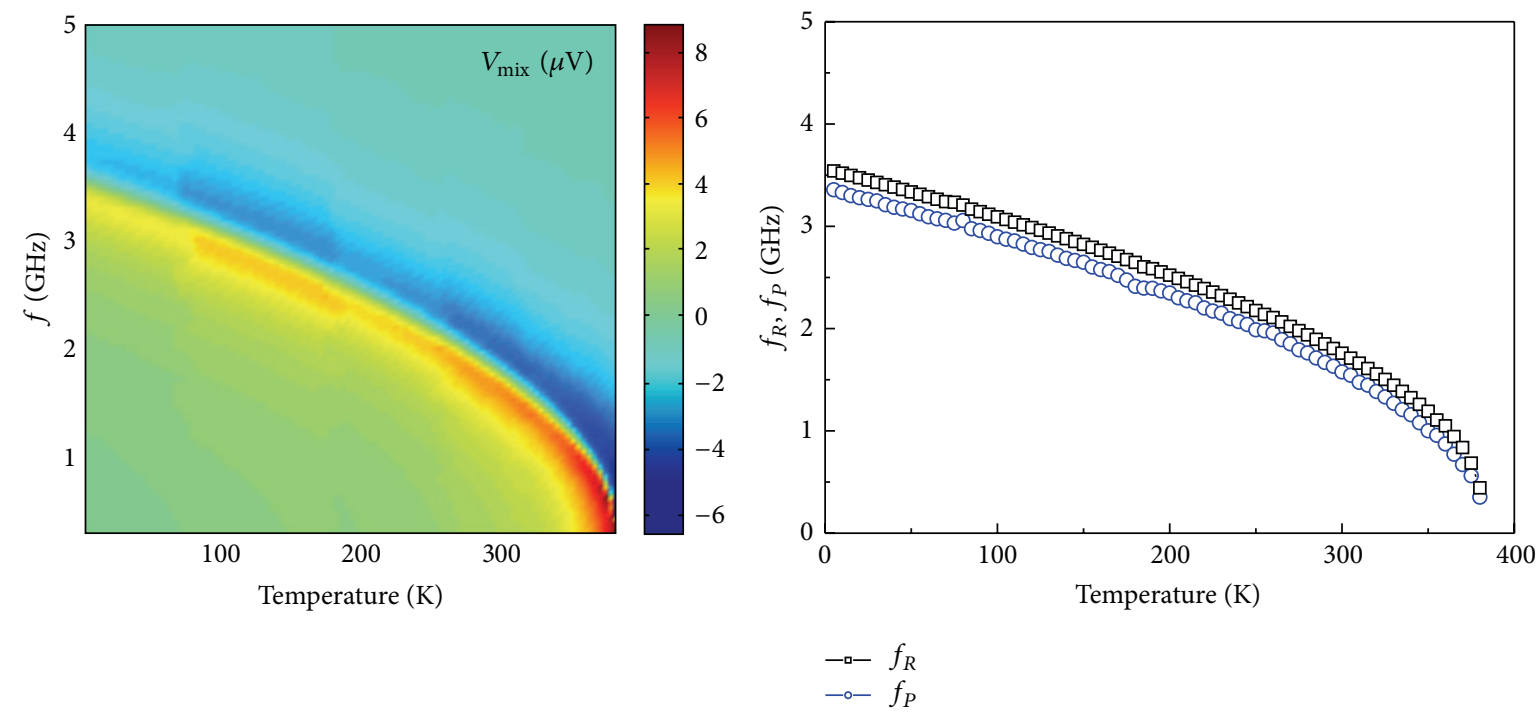

(a)
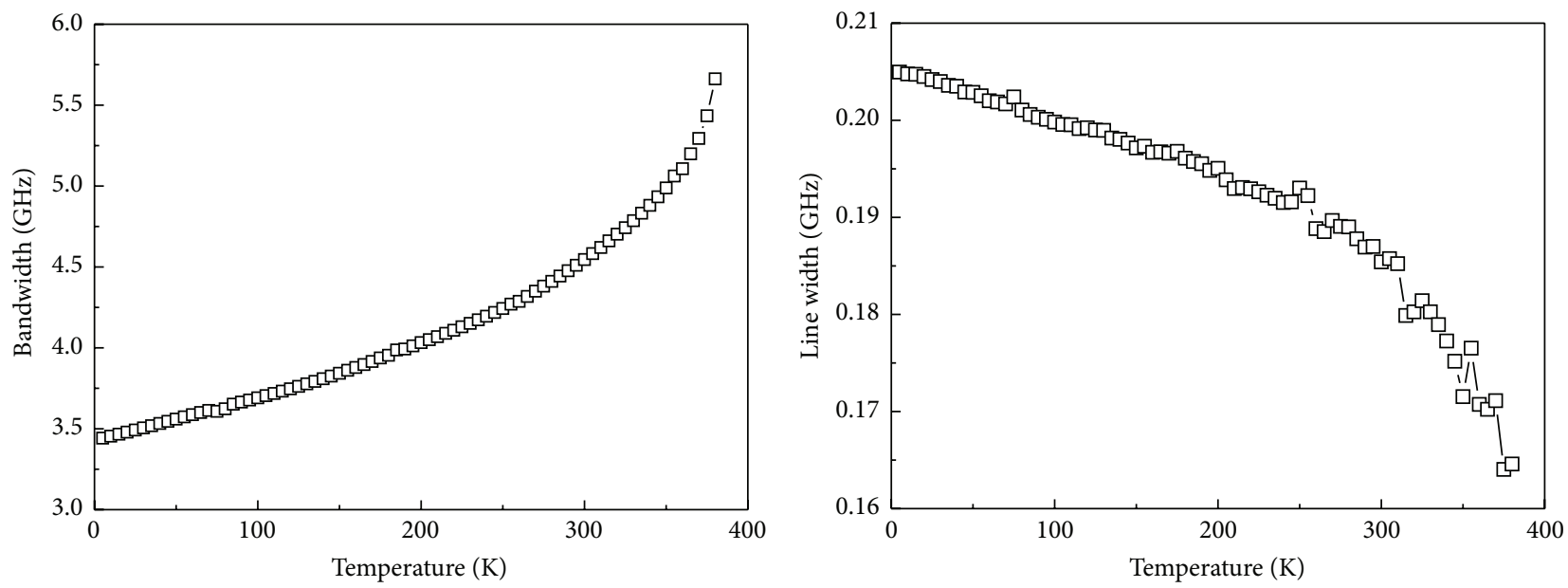

(c)

(d)

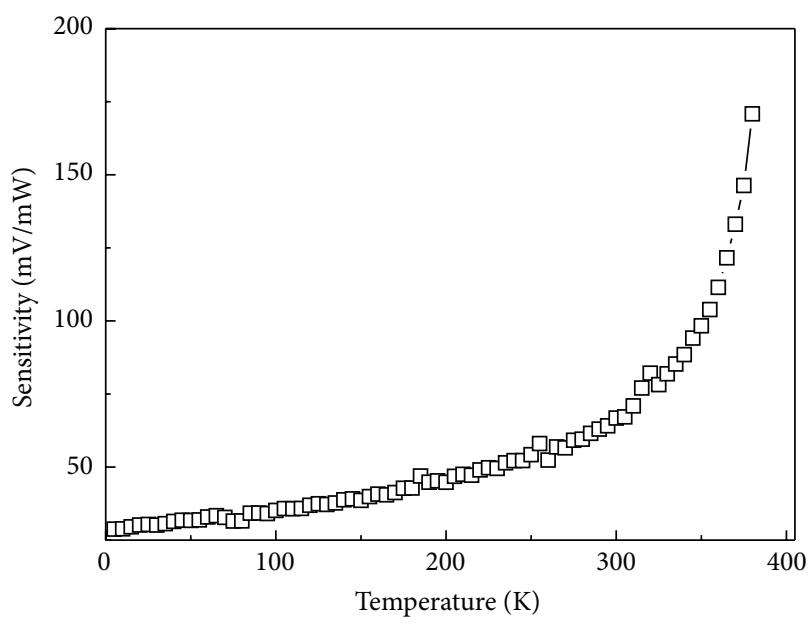

(e)

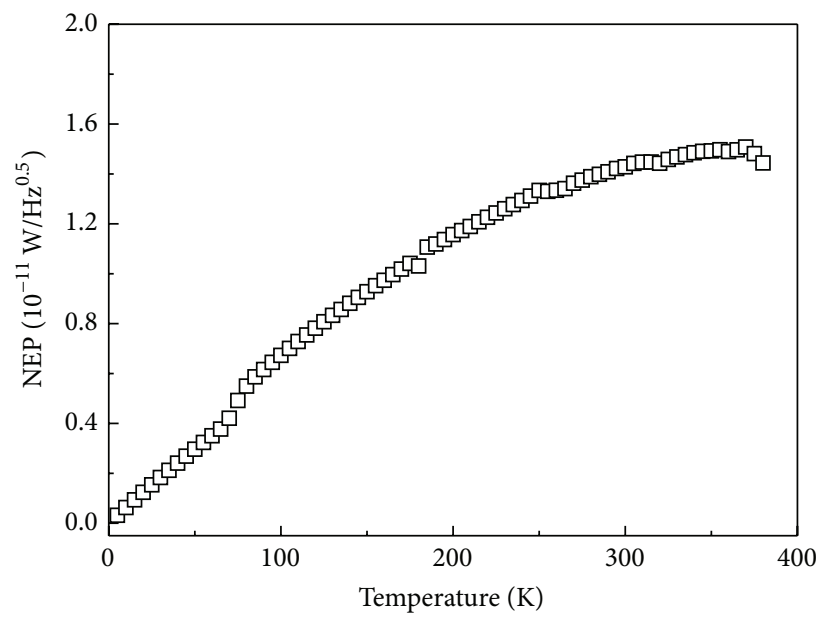

(f)

Figure 5: (a) The contour plots of $V_{\text {mix }}-f$ spectra, the temperature dependence of (b) $f_{R}$ and $f_{P}$, (c) bandwidth, (d) line-width, (e) sensitivity, and (f) NEP when $\beta_{\mathbf{M}}=45^{\circ}, \mu_{0} H=-60 \mathrm{mT}, \theta_{H}=90^{\circ}$, and $\delta_{H}=40^{\circ}$. 
provide insight for balancing these three performance parameters of STMDs through tuning the direction of magnetic field and the working temperature. The outcome of this work enables designing STMDs based on specific requirements on sensitivity, bandwidth, or NEP.

\section{Competing Interests}

The authors declare that they have no competing interests.

\section{Acknowledgments}

This work is supported in part by the Seed Funding Program for Basic Research and Small Project Funding Program from the University of Hong Kong, ITF Tier 3 funding (ITS/203/14, ITS/104/13, and ITS/214/14), RGC-GRF grant (HKU 17210014 and HKU 704911P), Innovation and Technology Fund Internship Programme (InP/182/14), and University Grants Committee of Hong Kong (Contract no. AoE/P-04/08).

\section{References}

[1] A. Fert, "Nobel lecture: origin, development, and future of spintronics," Reviews of Modern Physics, vol. 80, no. 4, pp. 15171530, 2008.

[2] P. A. Grünberg, "Nobel lecture: From spin waves to giant magnetoresistance and beyond," Reviews of Modern Physics, vol. 80, no. 4, pp. 1531-1540, 2008.

[3] E. B. Myers, D. C. Ralph, J. A. Katine, R. N. Louie, and R. A. Buhrman, "Current-induced switching of domains in magnetic multilayer devices," Science, vol. 285, no. 5429, pp. 867-870, 1999.

[4] J. Z. Sun, "Current-driven magnetic switching in manganite trilayer junctions," Journal of Magnetism and Magnetic Materials, vol. 202, no. 1, pp. 157-162, 1999.

[5] J. Z. Sun and D. C. Ralph, "Magnetoresistance and spin-transfer torque in magnetic tunnel junctions," Journal of Magnetism and Magnetic Materials, vol. 320, no. 7, pp. 1227-1237, 2008.

[6] D. C. Ralph, Y.-T. Cui, L. Q. Liu, T. Moriyama, C. Wang, and R. A. Buhrman, "Spin-transfer torque in nanoscale magnetic devices," Philosophical Transactions of the Royal Society A: Mathematical, Physical and Engineering Sciences, vol. 369, no. 1951, pp. 3617-3630, 2011.

[7] S. I. Klselev, J. C. Sankey, I. N. Krivorotov et al., "Microwave oscillations of a nanomagnet driven by a spin-polarized current," Nature, vol. 425, no. 6956, pp. 380-383, 2003.

[8] W. H. Rippard, M. R. Pufall, S. Kaka, S. E. Russek, and T. J. Silva, "Direct-current induced dynamics in $\mathrm{Co}_{90} \mathrm{Fe}_{10} / \mathrm{Ni}_{80} \mathrm{Fe}_{20}$ point contacts," Physical Review Letters, vol. 92, no. 2, Article ID 027201, 4 pages, 2004.

[9] A. A. Tulapurkar, Y. Suzuki, A. Fukushima et al., "Spin-torque diode effect in magnetic tunnel junctions," Nature, vol. 438, no. 7066, pp. 339-342, 2005.

[10] C. Wang, Y.-T. Cui, J. Z. Sun, J. A. Katine, R. A. Buhrman, and D. C. Ralph, "Sensitivity of spin-torque diodes for frequencytunable resonant microwave detection," Journal of Applied Physics, vol. 106, no. 5, Article ID 053905, 2009.

[11] C. Wang, Y.-T. Cui, J. Z. Sun, J. A. Katine, R. A. Buhrman, and D. C. Ralph, "Bias and angular dependence of spin-transfer torque in magnetic tunnel junctions," Physical Review B, vol. 79, no. 22, Article ID 224416, 2009.

[12] T. Taniguchi and H. Imamura, "Dependence of spin torque diode voltage on applied field direction," Journal of Applied Physics, vol. 114, no. 5, Article ID 053903, 2013.

[13] X. Li, C. Zheng, Y. Zhou, H. Kubota, S. Yuasa, and P. W. T. Pong, "Spin-torque diode with tunable sensitivity and bandwidth by out-of-plane magnetic field," Applied Physics Letters, vol. 108, no. 23, Article ID 232407, 2016.

[14] T. Taniguchi and H. Imamura, "Maximizing spin torque diode voltage by optimizing magnetization alignment," Applied Physics Express, vol. 6, no. 5, Article ID 053002, 2013.

[15] S. Miwa, S. Ishibashi, H. Tomita et al., "Highly sensitive nanoscale spin-torque diode," Nature Materials, vol. 13, no. 1, pp. 50-56, 2014.

[16] B. Fang, M. Carpentieri, X. Hao et al., "Giant spin-torque diode sensitivity in the absence of bias magnetic field," Nature Communications, vol. 7, p. 11259, 2016.

[17] T. Zeng, Y. Zhou, K. W. Lin, P. T. Lai, and P. W. T. Pong, "Spintorque diode-based radio-frequency detector by utilizing tilted fixed-layer magnetization and in-plane free-layer magnetization," IEEE Transactions on Magnetics, vol. 51, no. 11, 2015.

[18] O. V. Prokopenko, E. Bankowski, T. Meitzler, V. S. Tiberkevich, and A. N. Slavin, "Influence of temperature on the performance of a spin-torque microwave detector," IEEE Transactions on Magnetics, vol. 48, no. 11, pp. 3807-3810, 2012.

[19] J. Zhu, J. A. Katine, G. E. Rowlands et al., "Voltage-induced ferromagnetic resonance in magnetic tunnel junctions," Physical Review Letters, vol. 108, no. 19, Article ID 197203, 2012.

[20] J. Park, G. E. Rowlands, O. J. Lee, D. C. Ralph, and R. A. Buhrman, "Macrospin modeling of sub-ns pulse switching of perpendicularly magnetized free layer via spin-orbit torques for cryogenic memory applications," Applied Physics Letters, vol. 105, no. 10, Article ID 102404, 2014.

[21] J. C. Sankey, Y.-T. Cui, J. Z. Sun, J. C. Slonczewski, R. A. Buhrman, and D. C. Ralph, "Measurement of the spin-transfertorque vector in magnetic tunnel junctions," Nature Physics, vol. 4, no. 1, pp. 67-71, 2008.

[22] C. Kittel, "On the theory of ferromagnetic resonance absorption," Physical Review, vol. 73, no. 2, pp. 155-161, 1948.

[23] C. Kittel, "Interpretation of anomalous larmor frequencies in ferromagnetic resonance experiment," Physical Review, vol. 71, no. 4, pp. 270-271, 1947.

[24] S. Ishibashi, K. Ando, T. Seki et al., "High spin-torque diode sensitivity in $\mathrm{CoFeB} / \mathrm{MgO} / \mathrm{CoFeB}$ magnetic tunnel junctions under DC bias currents," IEEE Transactions on Magnetics, vol. 47, no. 10, pp. 3373-3376, 2011.

[25] Z. Zeng, K. H. Cheung, H. W. Jiang et al., "Evolution of spinwave modes in magnetic tunnel junction nanopillars," Physical Review B-Condensed Matter and Materials Physics, vol. 82, no. 10, Article ID 100410, 2010.

[26] B. Carvello, C. Ducruet, B. Rodmacq et al., "Sizable roomtemperature magnetoresistance in cobalt based magnetic tunnel junctions with out-of-plane anisotropy," Applied Physics Letters, vol. 92, no. 10, Article ID 102508, 2008.

[27] T. Moriyama, T. J. Gudmundsen, P. Y. Huang et al., “Tunnel magnetoresistance and spin torque switching in MgO-based magnetic tunnel junctions with a Co/Ni multilayer electrode," Applied Physics Letters, vol. 97, no. 7, Article ID 072513, 2010.

[28] W. X. Wang, Y. Yang, H. Naganuma, Y. Ando, R. C. Yu, and X. F. Han, "The perpendicular anisotropy of $\mathrm{Co}_{40} \mathrm{Fe}_{40} \mathrm{~B}_{20}$ 
sandwiched between $\mathrm{Ta}$ and $\mathrm{MgO}$ layers and its application in $\mathrm{CoFeB} / \mathrm{MgO} / \mathrm{CoFeB}$ tunnel junction," Applied Physics Letters, vol. 99, no. 1, Article ID 012502, 2011.

[29] Y. Zhou, C. L. Zha, S. Bonetti, J. Persson, and J. Åkerman, "Microwave generation of tilted-polarizer spin torque oscillator," Journal of Applied Physics, vol. 105, no. 7, Article ID 07D116, 2009.

[30] Y. Zhou, C. L. Zha, S. Bonetti, J. Persson, and J. Åkerman, "Spintorque oscillator with tilted fixed layer magnetization," Applied Physics Letters, vol. 92, no. 26, Article ID 262508, 2008. 

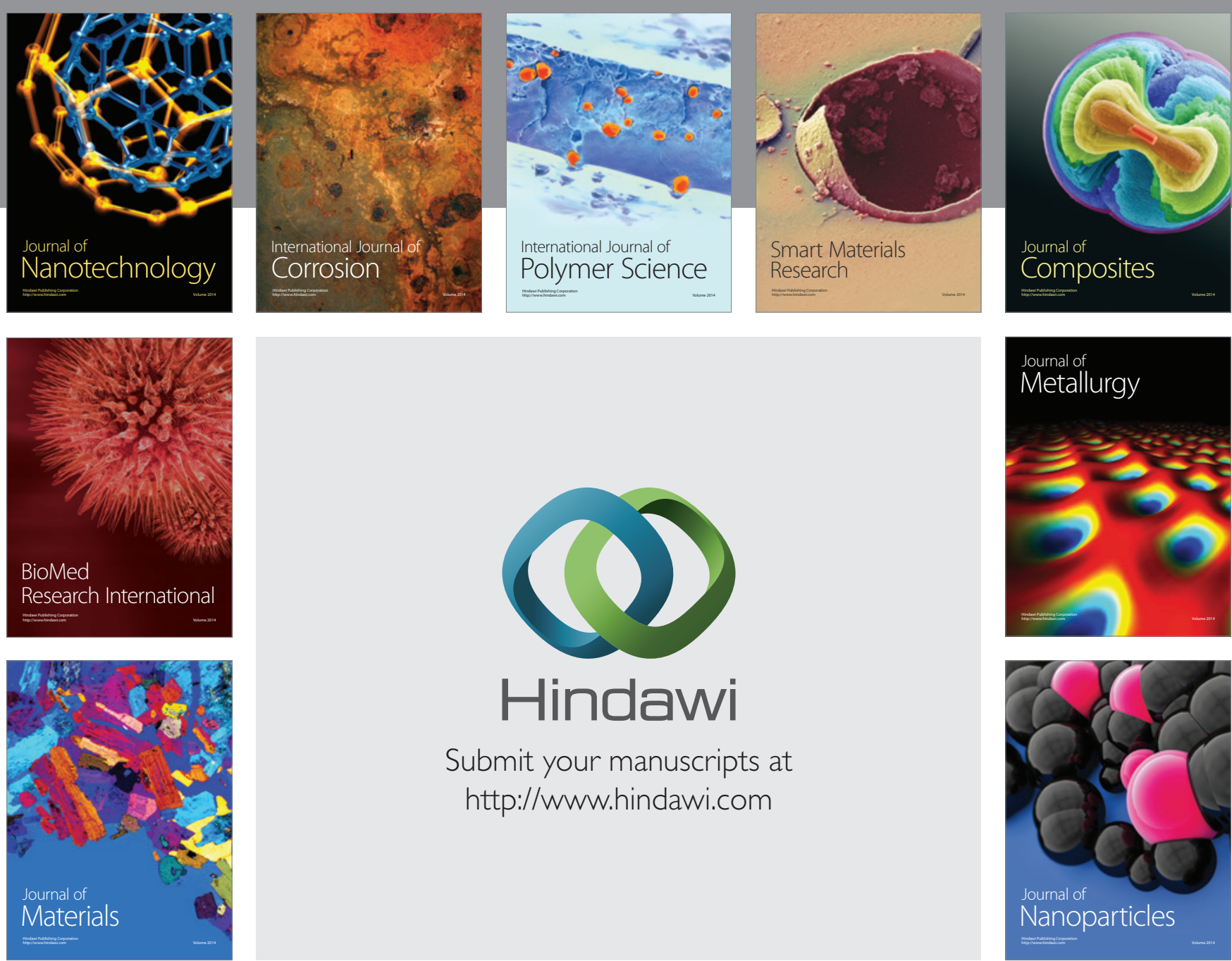

\section{Hindawi}

Submit your manuscripts at

http://www.hindawi.com

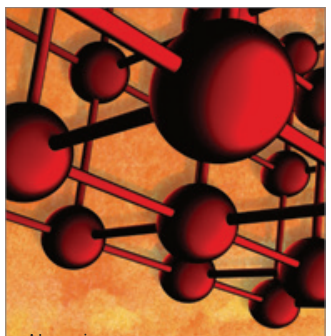

Materials Science and Engineering
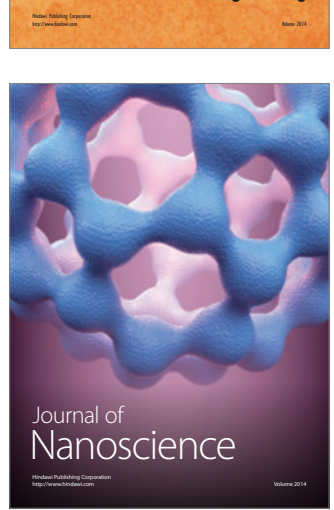
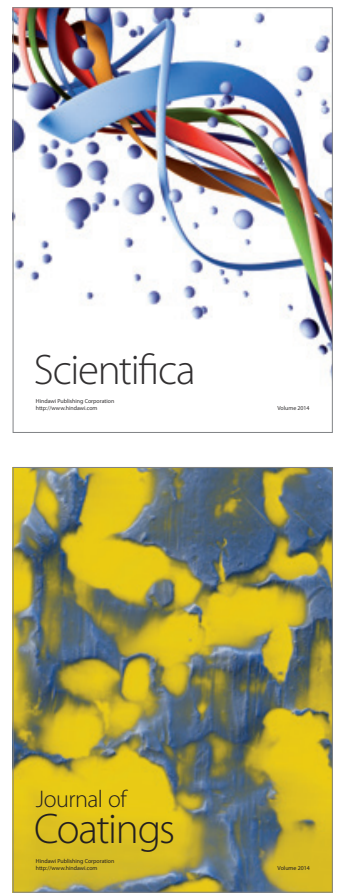
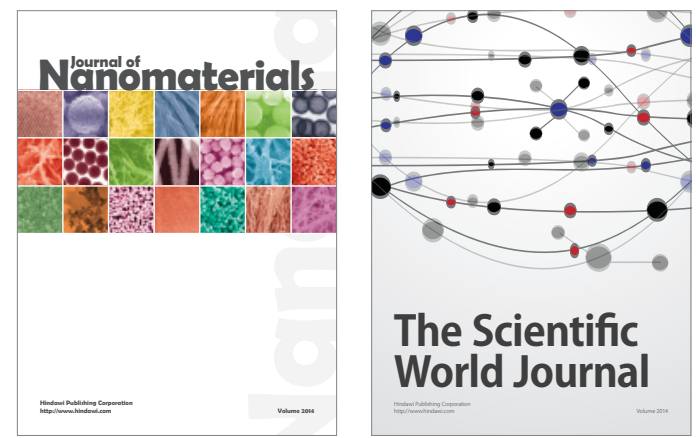

The Scientific World Journal
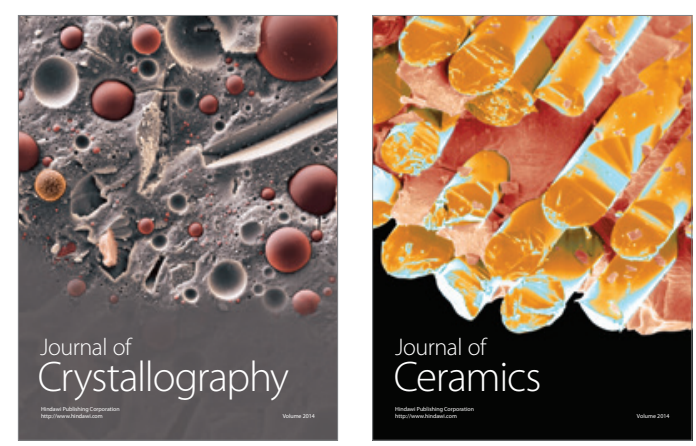
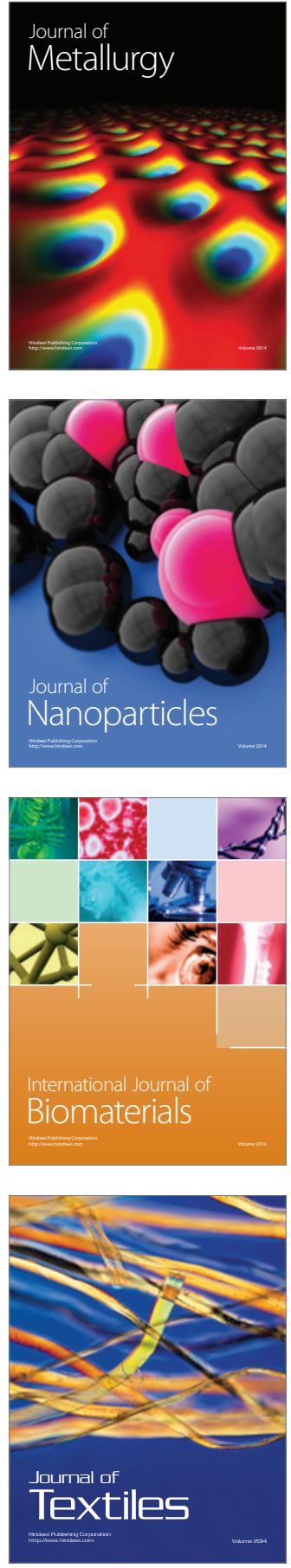